\title{
Utilization of Bio-Signals to Understand the Physiological States of e-Learners and Improve the Learning Contents
}

\author{
C. M. Althaff IRFAN ${ }^{\dagger a}$, Shusaku NOMURA ${ }^{\dagger}$, Takaoi YAMAGISHI ${ }^{\dagger}$, Yoshimasa KUROSAWA ${ }^{\dagger}$, \\ Kuniaki YAJIMA $^{\dagger \dagger}$, Nonmembers, Katsuko T. NAKAHIRA ${ }^{\dagger}$, Member, Nobuyuki OGAWA ${ }^{\dagger \dagger \dagger}$, Nonmember, \\ and Yoshimi FUKUMURA ${ }^{\dagger}$, Member
}

\begin{abstract}
SUMMARY This paper presents a new dimension in e-learning by collecting and analyzing physiological data during real-world e-learning sessions. Two different content materials, namely Interactive (IM) and Noninteractive (N-IM), were utilized to determine the physiological state of e-learners. Electrocardiogram (ECG) and Skin Conductance Level (SCL) were recorded continuously while learners experienced IM and N-IM for about 25 minutes each. Data from 18 students were collected for analysis. As a result significant difference between IM and N-IM was observed in SCL $(p<.01)$ meanwhile there were no significance in other indices such as heart rate and its variability, and skin conductance response (SCR). This study suggests a new path in understanding e-learners' physiological state with regard to different e-learning materials; the results of this study suggest a clear distinction in physiological states in the context of different learning materials.

key words: e-learning, skin conductance level, skin conductance response, ECG, heart rate variability
\end{abstract}

\section{Introduction}

E-learning is experiencing unprecedented growth and will continue to do so for the foreseeable future. A new analysis and forecast released by a research firm, showing that e-learning, reached by dollar volume \$27.1 billion in 2009 and predicting this figure will nearly double that by 2014 , with academic institutions leading the way [1]. On the other hand, bored students are dropping out of online classes while pleading for richer and more engaging online learning experiences [2], [3]. A well designed e-learning can increase retention over instructor-led training by up to $40 \%$ says the American Society for Training and Development-ASTD [1]. Given the importance of the e-learning, to provide an enhanced learning environment, it is crucial to understand the physiological phenomenon of the learners with regard to the presented materials or contents in the e-learning systems.

E-learning is a complex human learning phenomenon that a person needs to study its multiple facets from distinct angles. It is not only an educational phenomenon but also a complex phenomenon that involves cognitive, social, developmental, neurological, and other processes.

\footnotetext{
Manuscript received November 29, 2010.

Manuscript revised February 1, 2011.

${ }^{\dagger}$ The authors are with the Nagaoka University of Technology, Nagaoka-shi, 940-2188 Japan.

${ }^{\dagger}$ The author is with the Sendai National College of Technology, Sendai-shi, 989-3128 Japan.

t+t The author is with the Gifu National College of Technology, Motosu-shi, 501-0495 Japan.

a)E-mail: camirfan@oberon.nagaokaut.ac.jp

DOI: 10.1587/transinf.E94.D.1235
}

A vast number of attempts have been made on detecting human physiological states by employing a variety of physiological sensors. Electroencephalogram (EEG) and heart rate variability (HRV) have been frequently introduced to evaluate human psychophysiological states, while other bio-electric signals, such as skin temperature are recently integrated into the list of physiological measures [4], [5]. However only a few attempts have been made to investigate the physiological reactions of attendees of e-learning course [6], [7], and little is known about what types of learning materials induce what kind of physiological reactions on attendees or learners.

This study mainly focuses on the physiological changes in learners or students in the context of two different e-learning materials to ascertain physiological states of the learners during distinct learning materials with the use of different biosignals such as Electrocardiogram (ECG) and Skin Conductance Level (SCL).

Electrodermal activity (EDA) refers to variations in electrical conductance or its reciprocal, the resistance of the skin. These fluctuations occur as the result of the activity of the eccrine sweat glands, which are activated by sympathetic nervous system [8], [9]. This study utilizes the specific form of EDA, the Skin Conductance Level (SCL) [9], for the measurement of Skin Conductance (SC). The SCL was chosen for the reason that it is an indicator of overall autonomic activity [10]. Mainly, studies in the past have utilised Skin Conductance Response (SCR) to measure the EDA, however, the tonic changes in the SCL, such as those often recorded over longer periods (usually for minutes) and phasic changes often recorded in the form of SCR and for a shorter period (usually less than a minute in the presence of a discrete stimuli) may not precisely reflect the same underlying physiological states [11], [12]. SCR is utilized for measuring more transient changes in the SC. On the other hand, there are past studies, commending the use of SCL for the biofeedback [10], [13], however, these past studies failed to clearly indicate the application area and the benefits of using SCL over SCR.

The SC measurement has a greater advantage over other physiological signals of autonomic nervous system as it is under strict control of the sympathetic branch of the nervous system [14]. SC measurement provides readily accessible autonomic indices [14]. Further, heart rate (HR) and SC have been used to monitor task demand [15], [16]. SC and HRV have also been studied as general indicators of 
stress [17], [18]. The SC has also been used in important research on anxiety and stress levels [19]; and it has been a part of lie detection. Verschuere et al. (2004) reported an enhanced electrodermal response during the crime related picture viewing compared to the controlled picture viewing [9], [20].

Deviating from the general studies, the present study was conducted to assess the physiological state variability in subjects during the e-learning sessions. SC was utilized as the prime index in understanding the physiological state of the subjects for two different types of content materials namely interactive (IM) and non-interactive (N-IM) material. Further, high frequency (HF) of ECG is also utilized as it is an index for parasympathetic nervous system [21].

\section{Method}

\subsection{Subject}

Eighteen male students, age ranging from 19 to 20 years voluntary participated in this study. They were all domestic technical college students and majoring in Information Science and Technology.

\subsection{Procedure}

The subjects were instructed to attend two e-learning course materials; 1) a video lecture on advanced mathematics, and 2) a virtual laboratory work on electric circuits. These two course materials were provided by a proprietary e-learning system in our institute (e-learning Higher Education Linkage Project; eHELP), which is one of the largest effectively running e-learning project in Japan [22]-[24]. The lecture on advanced mathematics was a video-recorded material of the ordinary instructor led lecture, in which a university professor delivers a lecture in front of a video camera, so it was characterized as a non-interactive material $(N-I M)$. The other was a virtual laboratory work on electric circuits in which attendees were required to perform a number of given tasks with a terminal PC, by its nature, it was characterized as an interactive material (IM). All subjects participated in both N-IM and IM conditions (within-subjects experiment). Each material took roughly about $25-35$ minutes to complete.

More detailed description of the materials N-IM and IM are given in the next section.

\subsection{Materials}

The non-interactive material (N-IM) is a prerecorded video on advanced mathematics in which a professor appears and delivers a lecture on advanced mathematics theory with examples. This video is streamed online and played on an embedded video player inside the e-learning portal. The subjects are not required to perform any operations or manipulations on the e-learning portal, rather the subjects will only listen and watch the video of the lecture. Since this session of learning involves no interaction from the subject, it is classified as non-interactive material. The contents of this video lecture are solely on advanced mathematics. The professor explains the background theory and examples of advanced mathematical equations such as linear functions, quadratic functions, quartic functions and quintic functions and so on.

The interactive material (IM) is structured to be a virtual laboratory experiment on electric circuits. The virtual laboratory is a virtual environment on the e-learning portal where the subject can assemble and experiment different electronic circuits the same way he/she would do in a real life laboratory. The virtual laboratory is presented online inside the e-learning portal where the subject will manipulate and assemble a virtual electric circuit. Therefore, this session requires effort and brain work to assemble the circuits. Further, the subjects will use keyboard, mouse and other devices to make the circuits. This particular session was conducted to understand the principles operation of the Light Emitting Diode (LED), and virtual experiments on band theory and characteristic of current and voltage were also required to be designed. Further, referencing slide and video explanation on the real-laboratory experiment were also presented. The result of the virtual experiments on voltage characteristic is provided in the form CSV. Subjects are asked to derive an inner electrical resistance by analyzing this CSV.

Figure 3 and Fig. 4 are the screenshots of N-IM and IM.

\subsection{Measurements}

While the experiments were on-going the physiological measures (bio-electric signals) were recorded concurrently. EDA in terms of SCL, and Electrocardiogram (ECG) were recorded by bio-amplifier (BIOPAC MP150 systems, BIOPAC Systems Inc.), as shown in Fig. 1.

Figure 2 illustrates the theory behind the SCR and SCL, as shown in the figure the SCR are the more transient changes in SC while the SCL changes slowly.

SCL was recorded by the constant voltage method $(0.5 \mathrm{~V}), \mathrm{Ag}-\mathrm{AgCI}$ electrodes $(8 \mathrm{~mm}$ diameter active area)

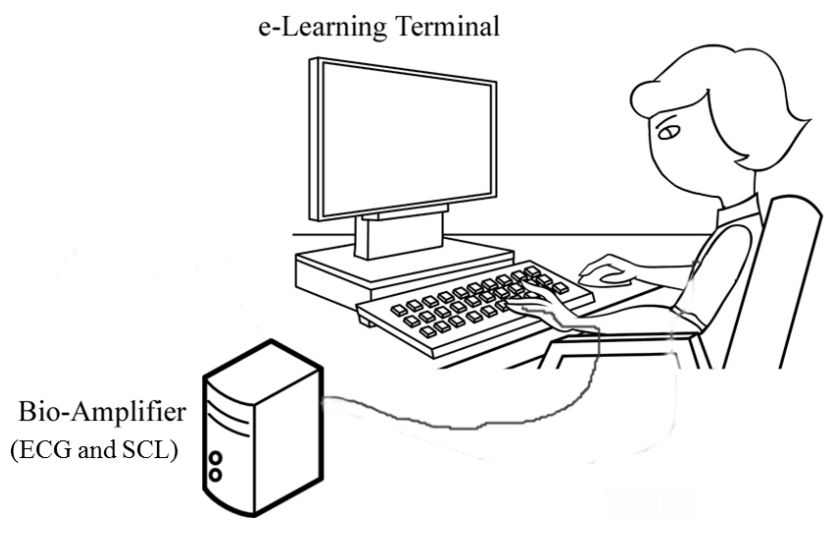

Fig. 1 Layout of experimental device. 


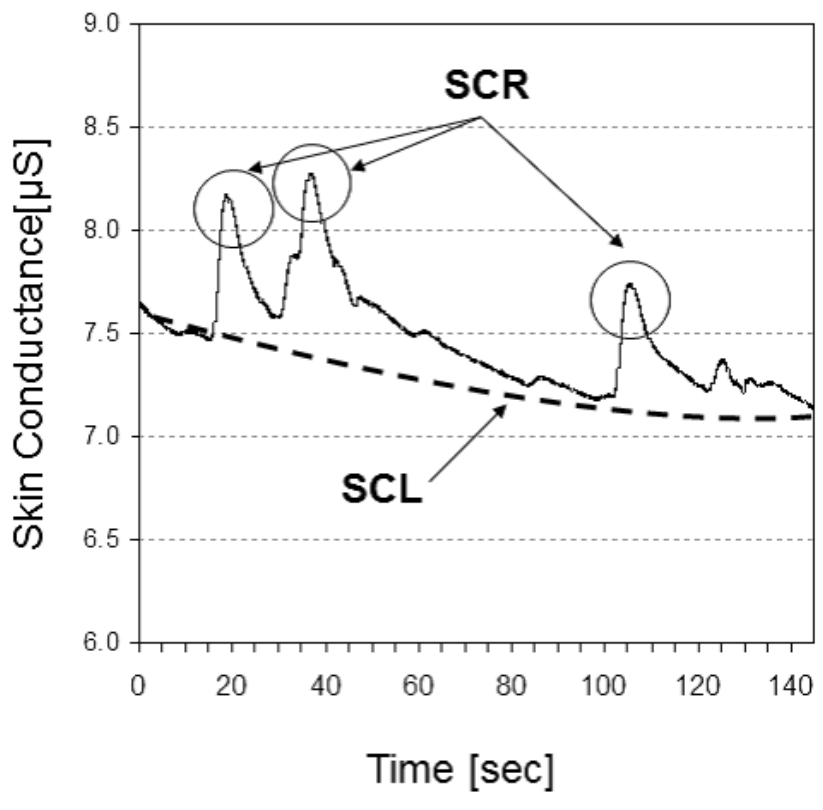

Fig. 2 Illustration of SCL and SCR.

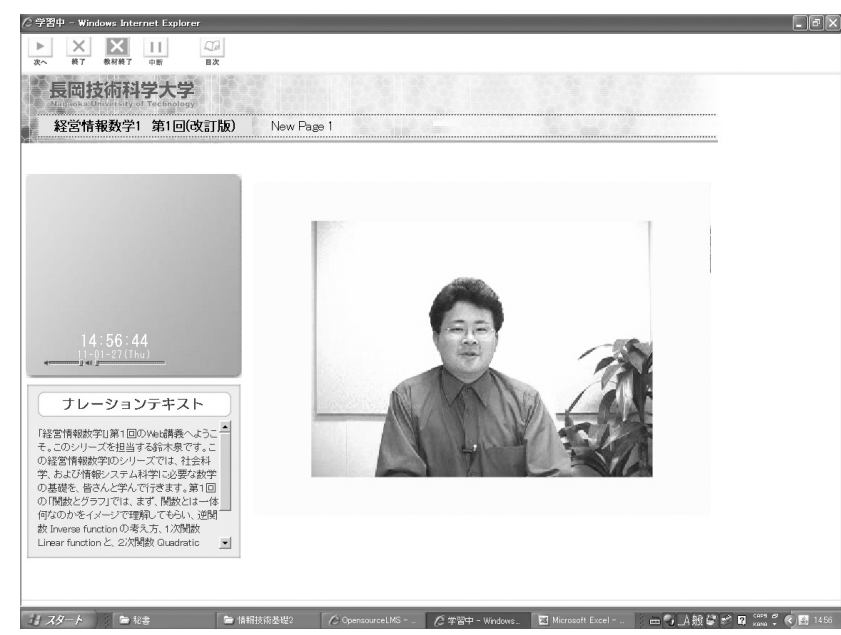

Fig. 3 Screenshot of non-interactive material.

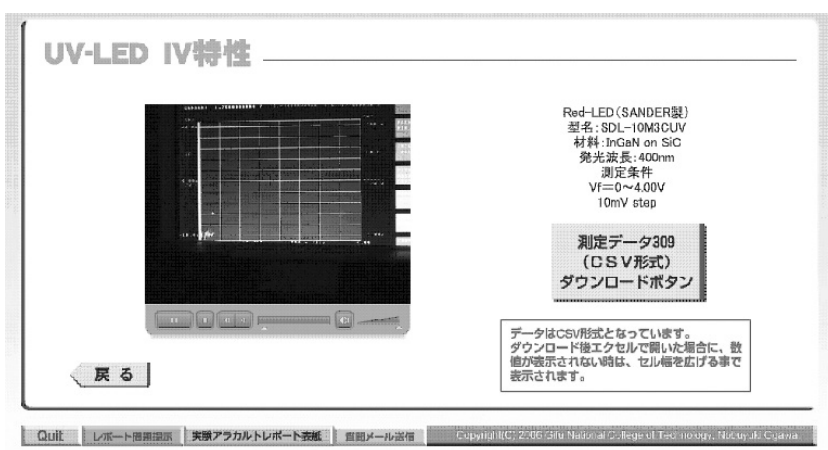

Fig. 4 Screenshot of interactive material.

filled with a skin conductance gel were attached to the palmer side of the middle phalanges of the second and third fingers of the subjects' non-dominant hand. Addi- tionally, for the purpose to illustrate the distinctive profile/characteristics of SCL, the incidence rate of SCR was calculated by counting the spikes in each minute epoch after treating the raw SCL data with high-pass filtering. ECG was recorded by placing the pre-gelled disposable electrodes. These physiological signals were taken continuously in time series at $200 \mathrm{~Hz}$ of sampling rate.

The skin conductance (SCR, SCL) has been used in a number of past studies, and the experimental results have been interpreted by these studies in many different ways i.e. anxiety, stress, nervous, dislike, curiosity etc. Therefore, we would like to suggest the interpretation of the experimental results of this particular study by reasoning and referring to the past studies.

\subsection{Data Analysis}

Since each material took roughly about 25-35 minutes to complete, we analyzed physiological signals of the first 25 minutes for the purpose of easy comparison. Frequency analysis was performed on ECG data to calculate High Frequency (HF) $(0.15-0.40 \mathrm{~Hz}$; HF) power of the signal. HF is considered to represent the parasympathetic nervous system activity [21], therefore it can be taken as an index of subjects' "relaxing" or "resting" sate. The collected physiological signals were segmented into every 60 seconds and averaged. Further, for the purpose of the better comparison of within-subject experimenting, the data in N-IM and IM conditions were normalized as follows:

$$
z_{i}=\frac{x_{i}-\bar{x}}{\sigma}
$$

where $z_{i}$ is the normalized value, and $x_{i}, \bar{x}$ and $\sigma$ are original value (an averaged value of a particular segment), averaged value, and standard deviation of the original value, respectively. In the statistical analysis, paired Students t-test was performed in comparing the differences between the conditions, and Wilcoxon signed-rand test was also performed in comparing the difference within the condition in order to test if certain values increased or decreased throughout the experiment period.

\section{Results}

Figure 5 (a) shows SCL profile in time series for N-IM and IM conditions, meanwhile Fig. 5 (b) represents the average change in SCL values throughout the experiment periods. In order for the better comparison, each trend in Fig. 5 (a) was baseline-corrected, by which all data were adjusted so that the initial value in each condition was set to be 0.0. SCL dropped moderately right after the experiment was started in IM condition, the decline in SCL continued gradually for the most period in IM condition, while there was no significant change in SCL values in N-IM condition.

This pattern resulted in a significant difference of decline in IM condition $(p<.01)$. Meanwhile there was no significant change in SCL throughout the session in N-IM 
(a)

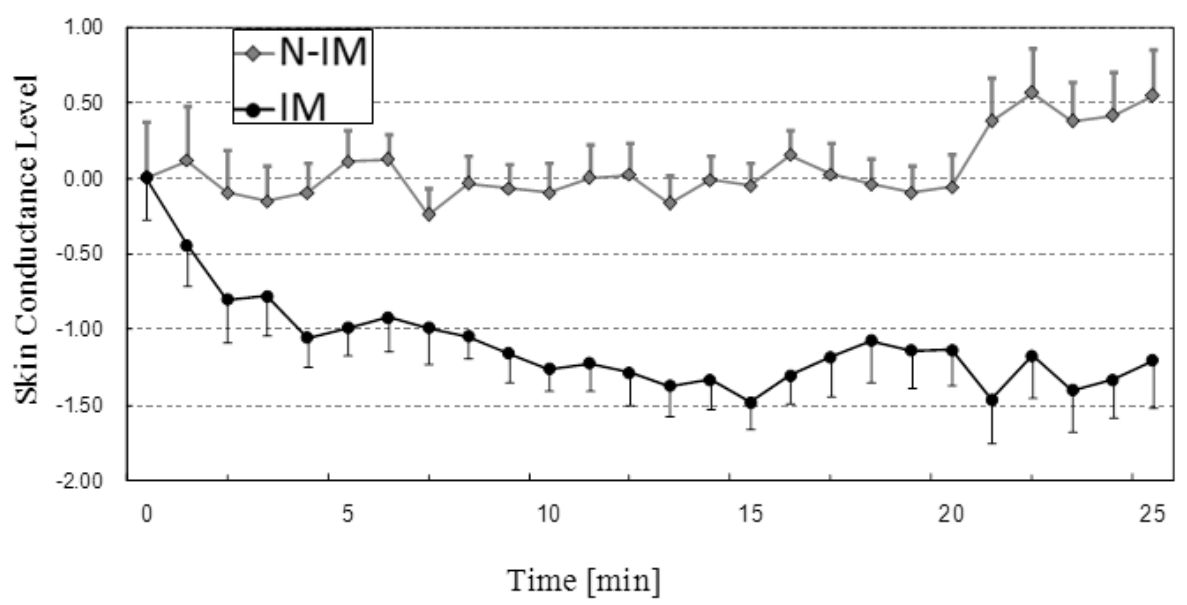

Fig. 5 (a) Profile of SCL and (b) overall average of SCL.

(a)

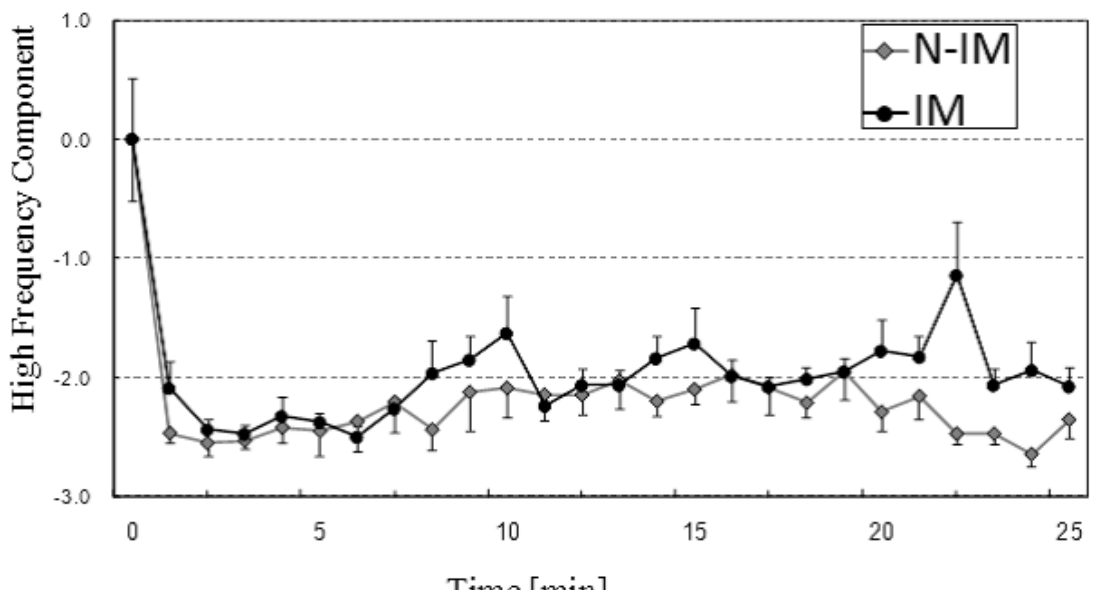

Time [min]

Fig. 6 (a) Profile of HF and (b) overall average of HF.

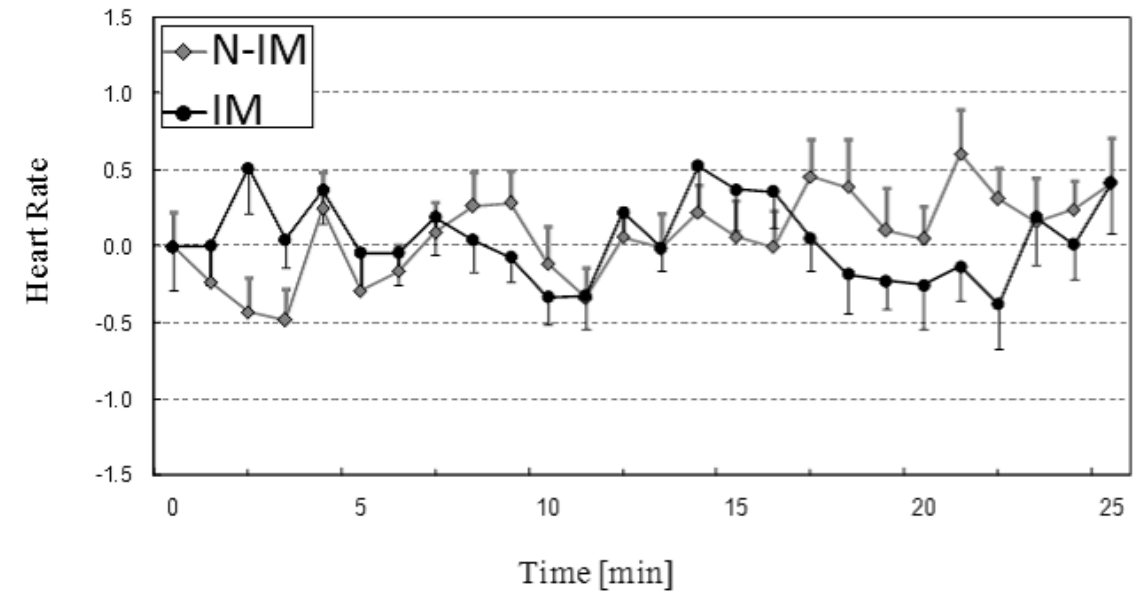

(b)

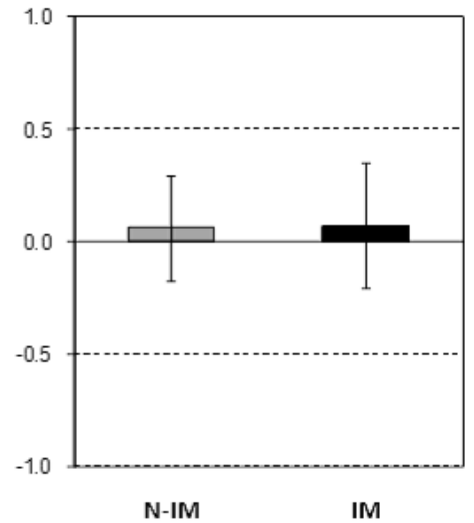

Fig. 7 (a) Profile of HR and (b) overall average of HR. 
(a)

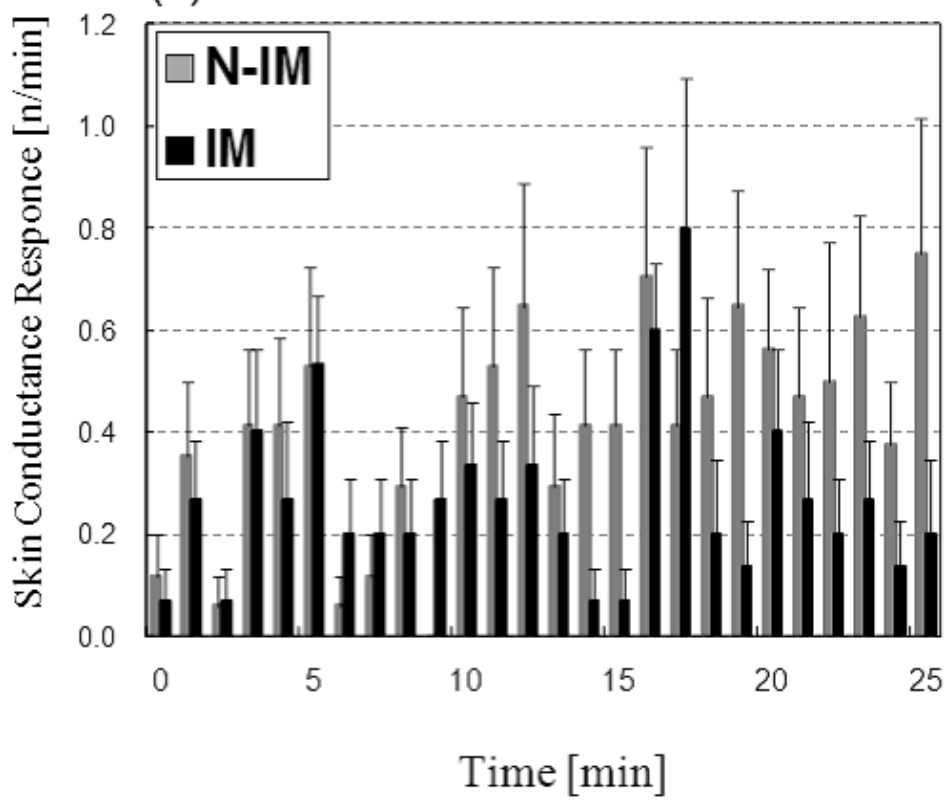

(b)

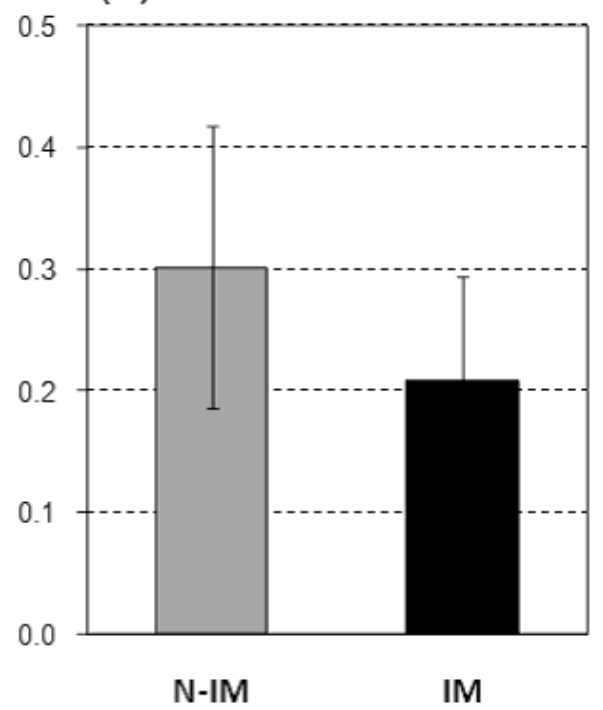

Fig. 8 (a) SCR occurrence rate in time series and (b) overall average of SCR occurrence rate.

condition $(p>.05)$, this resulted in a significant difference between IM and N-IM conditions $(p<.01)$.

Figure 6 (a) shows the HF profile in time series for N-IM and IM conditions; meanwhile Fig. 6(b) represents the average change in HF values throughout the experiment periods. HF dropped immediately after the experiment was started in IM and N-IM conditions. There was no remarkable change in HF values between IM and N-IM conditions after the initial decline. However, the HF in both conditions stood below the baseline for the most period of the experiment. This pattern resulted in a significant decline in $\mathrm{HF}$ during both IM and N-IM conditions $(p<.01)$. In the meanwhile there were no significant difference between IM and N-IM conditions $(p>.05)$. Figure 7 (a) represents the HR profile in IM and N-IM conditions; meanwhile Fig. 7 (b) represents the average change in HR values during the experiment periods. There was no statistical significance in both IM and N-IM conditions and between IM and N-IM conditions.

Figure 8 (a) represents the incidents rate of SCR in IM and N-IM conditions; meanwhile Fig. 8 (b) represents the average of the rate throughout the experiment periods. There was no statistical significance in both IM and N-IM conditions and between IM and N-IM conditions.

\section{Discussion}

This study was conducted to ascertain the physiological changes in candidates or students of e-Learning in the context of different e-learning materials. In order to understand the physiological states of the learners, this study utilized two different types of materials, characterized by its virtue as interactive material and non-Interactive material.
Table 1 Summary of physiological responses.

\begin{tabular}{cccc}
\hline & SCL & HF & HR \\
\hline IM & $-* *$ & $-*$ & \pm \\
(Interactive Material) & & & \\
N-IM & & & \\
(Non-interactive Material) & \pm & $-* *$ & \pm \\
\hline
\end{tabular}

The physiological responses and obtained statistical analysis results are summarized in Table 1, where "+", “-”, and " \pm " represent the trends of each profile that was observed in the time series. It should be noted that the significance is denoted with "*" or "**" in case if the responses were statistically significant at the level of $p<.05$ or $p<.01$, respectively.

The results of this study clearly show that the physiological state of the e-learners can be retrieved by analyzing the biosignals. The present study introduced distinct biosignals to capture the physiological states; the HR was introduced as it is considered to be an index of bodily activities [9]. However, the HR profiles did not show any significance in any of the conditions. The HF is considered as an index for parasympathetic nervous system activities [8], [9] however the HF stood low for both conditions, and showed no difference between both conditions, in the mean time the SCR also showed no significance. On the contrary the SCL showed a distinguishing significant difference between the conditions, this compelling results show that SCL is a more suitable than the other biosignals.

HF values dropped drastically just after the start of both conditions and stood low for the whole period of both sessions. It is natural as numbers of past studies illustrated that HF can be a feasible index for "relax" and "rest" [5], [15]. 
In other terms, HF could be an index to show if a subject is engaged in an activity or not but not how the subject is engaged as illustrated in interactive or non-interactive materials. Therefore the HF alone may not render a fruitful meaning with regard to the physiological states of the subjects. However, the combination of SCL and HF may precisely represent the underlying physiological state of learners over a long time span.

The results obtained in this study are very explanatory and intriguing, most significantly the SCL; The SCL resulted in statistically significant difference of decline in IM condition $(p<.01)$, and was lowered as shown in 5 . In the meanwhile there were no significant changes in SCL throughout the session in N-IM condition $(p>.05)$. The pattern of the SCL profile suggests that N-IM did not influence or impact the sympathetic nervous system of the elearning candidates. On the other hand a significant suppression of sympathetic nervous system activity was assumed in IM condition.

This SCL trait in IM does not necessarily represent the implication of lessened mental activity during the sessions of Interactive Material. A study, conducted on novice and expert pistol shooters shows that Pre-shot SCL of expert shooters was lower for the best shots [25]. Ikezuki et al. (1985) describes a significant decline in SCL during a mental calculation of numbers as a mental concentration [20]. The background knowledge and the results obtained through this study suggest that the decline in the SCL level during the IM condition may have been caused due to the concentration or deep involvement in the material itself.

SCL and SCR are varieties of the EDA, refers to variations in electrical resistance or its' reciprocal the conductance of the skin. These fluctuations are activated by the sympathetic nervous system [8], [9]. The EDA is therefore utilized as a reliable index of the sympathetic nervous system activation [14], [20], [26]. Significant changes in EDA for various discrete stimuli have been reported in many studies. In the past, studies have utilized SCR as the physiological measurement unit [14], [20], [27]. Moreover these studies were conducted to capture the spontaneous or phasic changes in the SC. However, other past studies show that SCR may not represent the precise physiological state due to its rapid phasic changes, when the experiment period is considerably longer [10], [13]. In the present study, SCL was used as the prime physiological measurement unit as for the advantages that it is an indicator of overall autonomic activity and SCL may precisely depict the slow changes in the physiological state [10], [12], [13], [28]. Further, the results of SCR in this study attributes to the claim of the advantage of using SCL since the SCR signals depicted no statistical difference between both condition and within conditions at all, nevertheless there has been a trend of lower number of spikes in SCR in IM condition.

The overall results of this study suggest that the transient changes in SC may not precisely depict the underlying psychological states, and that the SCL is a better candidate to capture the underlying physiological state when the ex- periment period is significantly longer.

In overall, this study could not derive any meaningful responses from HF, HR or the SCR all alone. However, in this study, the SCL is demonstrated to be the most affective physiological measurement in longer period of experiments. Further the SCL along with the HF shown to precisely represent the physiological states.

This study yielded very significant results nevertheless there were limitations. All eighteen subjects took part in this study were males. As the present study was conducted in a technical school, there was a scarcity for female subjects. Therefore the study could not authentically verify if the gender of the subjects may or may not have an impact on physiological measurement and therefore eventually on the overall results. In addition the variety of learning materials used was limited. In the e-learning stream, the learning contents are produced and delivered in a numerous forms such as the synergistic virtual-space contents, online contents, offline contents and so forth. However, the present study utilized not more than two materials, and the future studies may derive different results when other learning materials are regarded.

\section{Conclusion}

This study was conducted to ascertain the physiological changes in candidates or students of e-learning in the context of different e-learning materials. The results of this study are convincingly argue for the use of biosignals such as SCL and ECG to understand the underlying physiological state of the learners. Our results also suggest that the use of SCL over SCR to represent the physiological status of the learners is more suitable and appropriate. Further, the HF may not distinguish between the learning materials. Therefore the combination of SCL and HF may precisely represent the underlying physiological state of learners over a long time span.

This study paves a new path in understanding learners' physiological state with regard to different e-learning materials; the results of this study suggest a clear distinction in physiological states in the context of different learning materials. The results of this study also show that IM contents are more responsive than the N-IM contents. The insight into such physiological states in return may help in restructuring, improving and individualizing the learning materials in the future, and to make e-learning more effective. The study also suggests future inventions utilizing the SCL to be used in the e-Learning centers such as SCL sensor embedded mouse and other peripherals.

\section{Acknowledgements}

We would like to express our deepest appreciation of cooperation of all participants of this study from Sendai National College of Technology. 


\section{References}

[1] G.L.T.R. Snapshots, "2009-2014 us revenue forecasts for self-paced elearning products and services (in \$us millions)." http://www.ambientinsight.com/Reports/RevenueSnapshots.aspx, accessed July. 8. 2010.

[2] J. Bonk, "Online teaching in an online world (executive summary)," USDLA Journal, vol.16, no.1.

[3] K.J. Kim and C.J. Bonk, "The future of online teaching and learning in higher education," EDUCAUSE Quarterly Magazine, vol.29, no.4, pp.22-30, 2006.

[4] A. Nozawa and M. Uchida, "Characterization of preference for viscosity and fragrance of cosmetic emulsions by autonomous nervous system activity," Proc. ICROS-SICE, pp.2133-2136, 2009.

[5] J.A. Healey and R.W. Picard, "Detecting stress during real-world driving tasks using physiological sensors," IEEE Trans. Intelligent Transportation Systems, vol.6, no.2, pp.156-166, 2005.

[6] S. Scotti, M. Mauri, R. Barbieri, B. Jawad, S. Cerutti, L. Mainardi, E.N. Brown, and M. Villamira, "Automatic quantitative evaluation of emotions in e-learning applications," Proc. IEEE Eng. Med. Biol. Soc., pp.1359-1362, 2006.

[7] T. Itou, Y. Isomoto, Y. Miyahara, and H. Yamasaki, "Relation of intellectual activity to physiological and psychological change on the data measurement in studying," IEICE Technical Report ET200111, 2001.

[8] J.L. Andreassi, Psychophysiology Human behavior and Physiological Response fourth Edithion, Publishers-Mahwah, New Jersey, 2000.

[9] J.L. Andreassi, Psychophysiology Human Behavior and Physiological Response Fifth Edition, Publishers-Mahwah, New Jersey, 2009.

[10] A. Umezawa and A. Kurohara, "Comparison of skin conductance and skin potential as an index in electrodermal biofeedback studies," Japanese Society of Biofeedback Research, vol.21, pp.29-36, 1994.

[11] L.H. Miller and B.M. Shmavonian, "Replicability of gsr indeces as a function of stress and cognitive activity," J. Pers Soc. Psychol., vol.2, no.5, pp.753-756, 1965.

[12] C. Krumhansl, "An exploratory study of musical emotions and psychophysiology," Canadian Journal of Experimental Psychol., vol.51, no.4, pp.336-352, 1997.

[13] M. Ikezuki, K. Harano, and S. Yamaguchi, "A basic research of $\mathrm{scl}$ as an index of organic responses applicable to the biofeedback method," Japanese Society of Biofeedback Research, vol.12, pp.1622, 1985.

[14] S. Khalfa, P. Isabelle, B. Jean-Pierre, and R. Manon, "Event related skin conductance response to musical emotions in humans," Neuroscience Letters, vol.328, pp.145-149, 2002.

[15] G.F. Wilson, "An analysis of mental workload in pilots during flight using multiple psychophysiologic measures," International. Journal of. Aviation. Psychology, vol.12, no.1, pp.3-18, 2001.

[16] M.A. Bonner and G.F. Wilson, "Heart rate measures of flight test and evaluation," International. Journal of. Aviation. Psychology, vol.12, no.1, pp.63-77, 2001.

[17] C.M.A. van Ravenswaaij-arts, L.A.A. Kollee, J.C.W. Hopman, G.B.A. Stoelinga, and H.P. van Geijin, "Heart rate variability," Annals of Internal Medicine, vol.118, no.6, pp.436-447, 1993.

[18] W. Boucsein, Electrodermal Activity, NY: Plenum, New York, 1992.

[19] W. Fenz and S. Epstein, "Gradients of physiological arousal in parachitists," Psychosomatic Medicine, vol.29, pp.33-51, 1967.

[20] B. Verschuere, G. Crombez, A.D. Clercq, and E.H.W. Koster, "Automatic and behavioral responding to concealed information: Differentiating orienting and defensive responses," Psychophysiology, vol.41, pp.461-466, 2004.

[21] B. Pomeranz, R. Macaulay, M. Caudill, I. Kutz, D. Adam, D. Gordon, K.M. Kilborn, A.C. Barger, D.C. Shannon, R.J. Cohen, and H. Benson, "Assessment of autonomic functions in human by heart rate spectral analysis," American Journal of Physiology, vol.248, no.1, pp.151-153, 1985

[22] T. Yukawa, I. Suzuki, and Y. Fukumura, "A cross-lms chat system and its evaluation," Proc. ED-MEDIA, pp.1340-1345, 2009.

[23] K.T. Nakahira, S. Suzuki, and Y. Fukumura, "A method of enhancing motivation in e-learning including its evaluation," Proc. ICCE, pp.603-604, 2008.

[24] Y. Fukumura and K. Maruyama, "E-learning program for supporting to create safety and secure society (in japanese)," J. Multimedia Education Research, vol.12, no.2, pp.37-48, 2004.

[25] P. Tremayne and R.J. Barry, "Elite pistol shooters physiological patterning of best and worst shots," International Journal of Psychophysiology, vol.41, pp.19-29, 2001.

[26] J.J. Alvarsson, S. Wiens, and M.E. Nilsson, "Stress recovery during exposure to nature sound and environmental noise," International Journal of Environmental Research and Public Health, vol.7, no.3, pp.1036-1046, March 2010.

[27] M.M. Bradley, "Natural selective attention: Orienting and emotion," Psychophysiology, vol.46, pp.1-11, 2009.

[28] D.I. Radin, "Electrodermal presentiments of future emotions," J. Scientific Exploration, vol.18, no.2, pp.253-273, 2004.

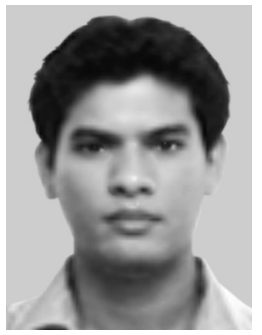

C. M. Althaff Irfan graduated and received his Masters degree from the London Metropoli$\tan$ University, UK. He is presently reading for his Ph.D. in Nagaoka University of Technology. $\mathrm{He}$ is also a member of the British Computer Society.

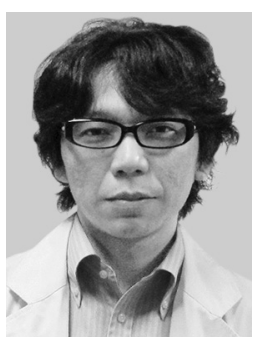

Shusaku Nomura is a Associate Professor in Nagaoka University of Technology, Nagaoka, Japan. He received the Diploma degree in Physics from Kobe University, Kobe, Japan in 1996, and the PhD in science from Kobe University Graduate School of Science and Technology, Kobe, Japan, in 2001.

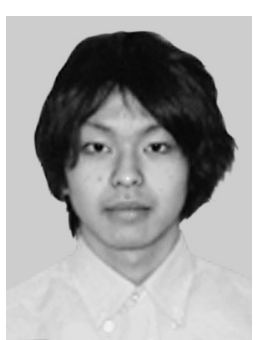

Takaoi Yamagishi graduated from Department of Management Information and Control Engineering in Nagaoka University of Technology. He is now reading for his master degree in physiological measurement. 


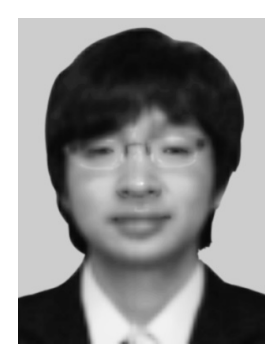

Yoshimasa Kurosawa graduated from Department of Management Information and Control Engineering in Nagaoka University of Technology. He is now reading for his master degree in physiological measurement.
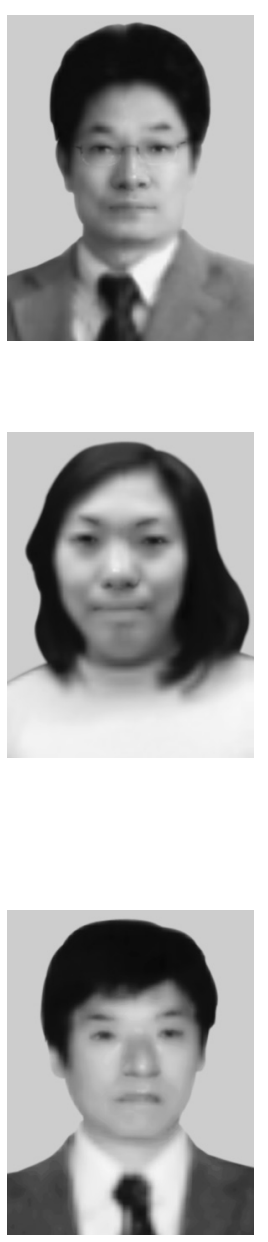

Nobuyuki Ogawa graduated from the Faculty of Engineering in Tsukuba University, and consequently received his $\mathrm{PhD}$ in Engineering. $\mathrm{He}$ is now with Gifu national College of Technology.

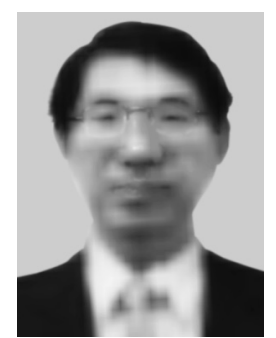

Yoshimi Fukumura is currently a professor of Management and Information Systems Engineering, and a head of Center for e-Learning Research and Application, Nagaoka University of Technology, Japan. His research interest is in educational technology and systems. He graduated from Kyoto University in 1973 and received Ph.D. from Nagoya Institute of Technology in 1996. He is a member of IEEE, and IPSJ. 